\title{
Robot-assisted laparoscopic retroperitoneal leiomyosarcoma resection with inferior vena cava graft replacement: a case report
}

\author{
Gong Cheng ${ }^{1,2 \#}$, Hailong Ruan ${ }^{1,2 \#}$, Chao Yang ${ }^{3}$, Qi Cao ${ }^{1,2}$, Huageng Liang ${ }^{1,2}$, Xiong Yang ${ }^{1,2}$, \\ Guosong Jiang $^{1,2}$, Xiaoping Zhang $^{1,2}$ \\ ${ }^{1}$ Department of Urology, Union Hospital, Tongji Medical College, Huazhong University of Science and Technology, Wuhan, China; ${ }^{2}$ Institute \\ of Urologic Surgery, Union Hospital, Tongji Medical College, Huazhong University of Science and Technology, Wuhan, China; ${ }^{3}$ Department of \\ Vascular Surgery, Union Hospital, Tongji Medical College, Huazhong University of Science and Technology, Wuhan, China \\ \#These authors contributed equally to this work. \\ Correspondence to: Xiaoping Zhang, Guosong Jiang. 1277, Jiefang Avenue, Wuhan 430022, China. Email: xzhang@hust.edu.cn, jgs017@126.com.
}

\begin{abstract}
The aim of the present study was to report the initial clinical experience of robot-assisted laparoscopic retroperitoneal leiomyosarcoma resection with inferior vena cava graft replacement. The patient was a 45 -year-old female with abdominal pain. She was referred to our hospital and found to be with a retroperitoneal mass $(46 \mathrm{~mm} \times 45 \mathrm{~mm})$. The inferior vena cava and the distal part of left renal vein were invaded by the tumor and compression was obviously seen from magnetic resonance imaging. The serum level of potassium, epinephrine, norepinephrine, cortisol, adrenocorticotropic hormone and renin angiotensin aldosterone system were all in normal ranges before the surgery. The operation was performed via a six port, robot assisted, transperitoneal laparoscopic approach. The tumor was completely resected and adherent part of inferior vena cava (approximately $5 \mathrm{~cm}$ ) was dissected. Considering severe impairment of the great vessel, we decided to replace excised caval segment with an extended polytetrafluoroethylene graft and undertook the inferior vena cava reconstruction. The patient was discharged 11 days postoperatively with embolus in the graft. Anticoagulants were routinely administrated and the thrombus seemed to be smaller 3 months after operation. Abdominal pain was resolved and pathological examination finally confirmed that the tumor was leiomyosarcoma with negative margins free from tumor. Leiomyosarcoma of inferior vena cava present a technical challenge to surgeons. Comprehensive preparation should be made preoperatively to facilitate tumor resection and vascular management. In specific cases, robotic resection of leiomyosarcoma from great vessels and vascular repairment might be feasible options in experienced hands.
\end{abstract}

Keywords: Robot; reconstruction; inferior vena cava

Submitted Dec 27, 2020. Accepted for publication Mar 04, 2021.

doi: $10.21037 /$ tau-20-1523

View this article at: http://dx.doi.org/10.21037/tau-20-1523

\section{Introduction}

Leiomyosarcomas (LMS) represent $5-10 \%$ of soft tissue sarcomas and are more frequent in women, which could be influenced by pregnancy and estrogenic stimulation (1). Originating from the vascular bed, LMS are more commonly in the great vessels such as the inferior vena cava (IVC) and renal veins (2). Adjuvant chemotherapy, adjuvant radiotherapy and surgeries are all possible choices for leiomyosarcomas of IVC, but surgeries are the only potentially curative therapies and now the mainstay of treatments (3). Surgically negative margins are associated with improved survival rates, but no consensus exists about the necessity for great vessel reconstruction (4). Traditional laparoscopic operation of tumors near great vessels is challenging due to limitation of two-dimensional vision and range of motion. Robotic surgical systems ( $d a$ Vinci Si ${ }^{H D}$ ) have been adopted universally recent years, due to the three-dimensional vision, enhanced ergonomics and minimally invasive surgery (5). The retroperitoneal tumor near great vessels usually makes 

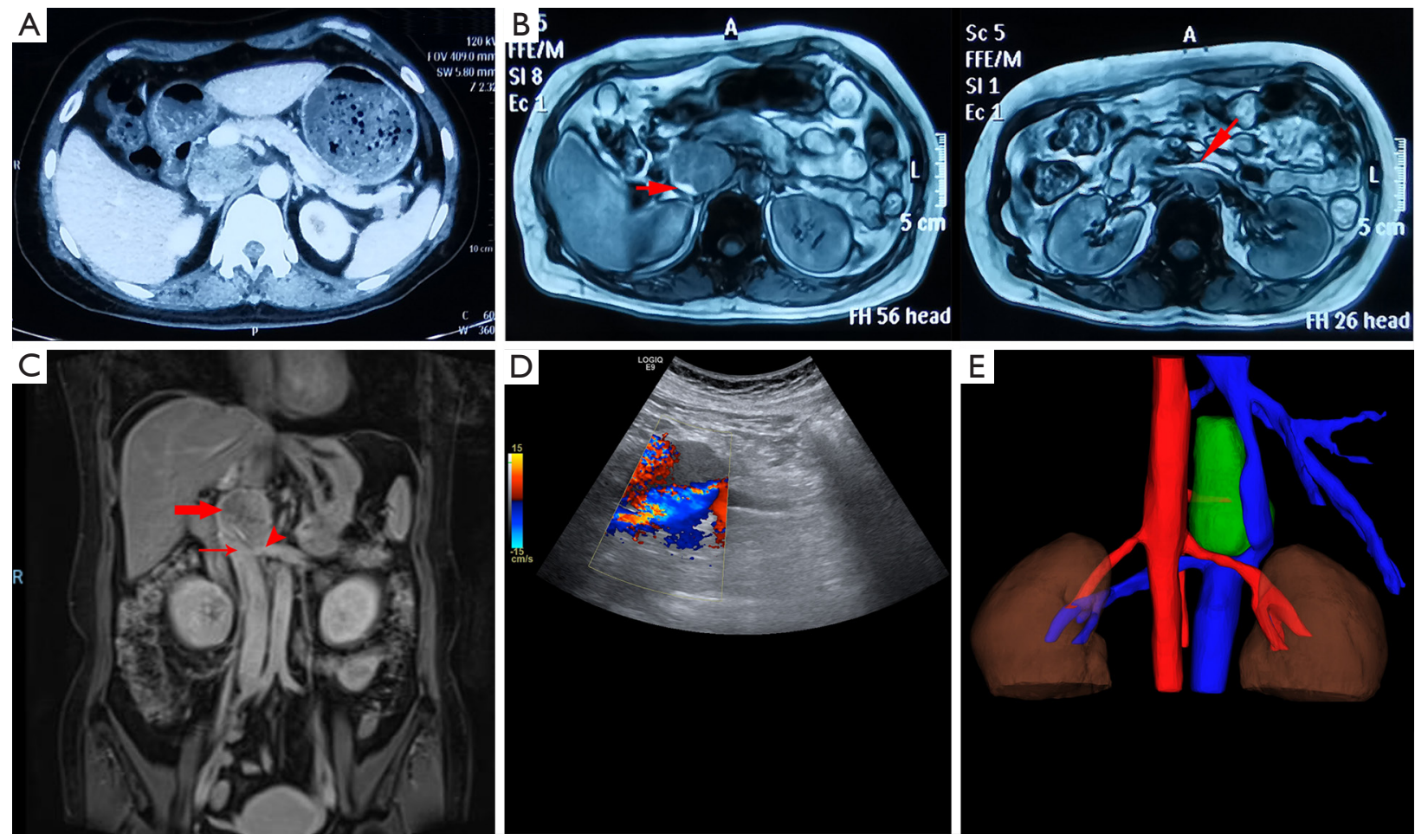

Figure 1 The tumor was assessed by imaging study. (A) CT scan indicated the tumor was near right adrenal gland and IVC. (B) MRI showed occlusion of inferior vena cava (left arrow) and local compression of left renal vein (right arrow). (C) An enhanced MRI was performed again to assess the tumor size (broad arrow) and tumor invasion of IVC (slim arrow) and left renal vein (short arrow). (D) Ultrasound indicated tumor invasion of IVC and local left renal vein. (E) Three-dimensional reconstruction of MRI image explicitly revealed tumor position (green) between veins (blue) and arteries (red) from the posterior direction. CT, computed tomography; MRI, magnetic resonance imaging; IVC, inferior vena cava.

more demand for wide exposure and retraction of peritoneal organs. Therefore, robot-assisted laparoscopic surgeries facilitate the minimally invasive resection of tumors and seem to be the solution for these problems.

Up till now, a series of cases have been reported about robot-assisted laparoscopic resection of LMS from venous systems, but no vascular reconstruction has been performed (6-9). In this case, we present the first described report of robotic resection of retroperitoneal LMS with IVC reconstruction. The study received institutional review board approval and written informed consent was obtained from the patient. We also presented the following case in accordance with the CARE reporting checklist (available at http://dx.doi.org/10.21037/tau-20-1523).

\section{Case presentation}

In January 2020, a 45-year-old woman $(165 \mathrm{~cm}, 55 \mathrm{~kg})$ sought local medical advices for right abdominal pain and was referred to our tertiary care center in April. Vital signs were normal and no other symptoms appeared. The patient had no cardiac diseases history and blood pressure was monitored daily to be under control.

In January 2020, an enhanced computed tomography (CT) scan in the local hospital identified a $46 \mathrm{~mm} \times$ $45 \mathrm{~mm}$ retroperitoneal mass near right adrenal gland, right renal vein and IVC (Figure 1A). Magnetic resonance imaging (MRI) further indicated that IVC was obviously occluded and the left renal vein was locally compressed (Figure 1B). The serum level of potassium, epinephrine, norepinephrine, cortisol, adrenocorticotropic hormone and renin angiotensin aldosterone system were all in normal ranges (data not shown). The glomerular filtration rate (GFR) was also normal $(115.9 \mathrm{~mL} / \mathrm{min})$ preoperatively. In April 2020, an enhanced MRI and ultrasound in our care center were performed respectively again to assess the 


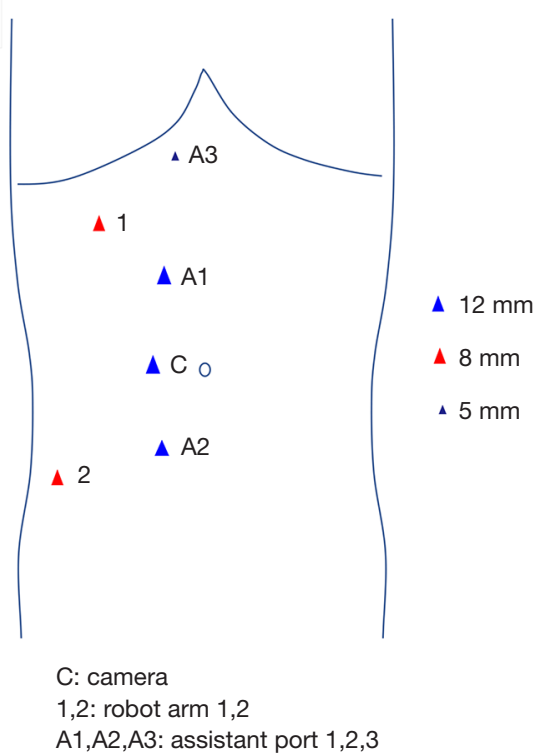

Figure 2 Trocar placement for the surgery. A six-trocar technique was applied for the surgery. One $12 \mathrm{~mm}$ trocar near the umbilicus was the designated camera port (point $\mathrm{C}$ ). Camera port, robot arm port 1 (near the liver margin) and 2 (above the anterior superior iliac spine) formed approximate 120 angular degree. Assistant port A1 and A2 were $8 \mathrm{~cm}$ from the camera port. A $5 \mathrm{~mm}$ trocar under the liver margin near the midclavicular line was used as the assistant port for liver retraction (point A3).

features of the mass including local tumor invasion, tumor size and IVC wall infiltration (Figure 1C,D). It was reported that the porta hepatis and pancreas were slightly pressed upward by the mass and the right renal artery was pushed downward. IVC and the distal part of left renal vein were invaded by the tumor and compression was obviously seen from MRI imaging. Three-dimensional reconstruction was also conducted to explicitly reveal tumor position and vessel shifts from the posterior direction (Figure 1E).

During the operation, the patient was placed in the left lateral decubitus position (with the right side elevated $\sim 70^{\circ}$ ) under general anesthesia and monitored by continuous central venous and arterial pressure monitoring. A six-port approach was used to insert the trocars (Figure 2). After pneumoperitoneum establishment, a robotic camera was inserted to examine adhesion of bowels and whether there were obvious indications against performing the surgery. The mass was dissected carefully from adhesive adipose tissues and the tumor was then exposed to our sight (Figure 3A). The fascia was circumferentially dissected from the tumor and vessels were then exposed. $8 \mathrm{Fr}$ red-rubber catheters were wrapped twice around the cephalic IVC, left renal vein (LRV), caudal IVC and right renal vein (RRV) sequentially and clamped by Hem-o-lok clips (Figure 3B). The tumor was completely resected and adherent part of IVC (approximately $5 \mathrm{~cm}$ ) was dissected (Figure 3C). Considering severe impairment of IVC, we decided to replace excised caval segment with a synthetic graft in an end-to-end fashion. Interposition graft is accepted when there exists en bloc IVC resection and extended polytetrafluoroethylene (ePTFE) graft is the preferred material as it has a high reported patency rate. After heparinization, the graft was placed between the cephalic IVC and caudal IVC in an end-to-end fashion. The RRV was also be included in the reconstruction by jointing its free end to the interposition graft (Figure 3D). Due to the continuity of the graft, the remaining LRV was clamped by Hem-o-lok clips and isolated with ligation (Figure $3 E$ ). An omental pedicle was fashioned to cover the synthetic graft and prevent graft to contact with bowels (Figure $3 F$ ). The tumor was placed in a specimen bag and the specimen subsequently underwent pathological examination.

The operative time was 9 hours 45 minutes and the IVC clamp proportion was 6 hours 50 minutes. The estimated blood loss was about 2,000 $\mathrm{mL}$ and the patient was transfused blood plasma with $600 \mathrm{~mL}$ intraoperatively. Anticoagulants (low molecular weight heparin, LMWH) were routinely subcutaneously injected with 4,000 units twice a day after operation. Lower extremity edema transiently occurred and it disappeared one week after the surgery. During hospitalization, the patient was asked to make an ultrasound and enhanced MRI examination one week postoperatively. The ultrasound indicated that there was a $45-\mathrm{mm}$ filling defection in the upper synthetic graft, which was in accordance with D-dimer $2.97 \mathrm{mg} / \mathrm{L}$ (Figure $4 A$ ). The blood flow velocity of renal artery was 78 (left) and $81.9 \mathrm{~cm} / \mathrm{s}$ (right) respectively (Figure 4B). Threedimensional reconstruction of MRI revealed that the graft was partially occluded by thrombus and we thought that venous return may flow through lumbar vein to hemiazygos vein system (Figure 4C). The continuity of right renal artery imaging was interrupted and signaling of the right kidney was weaker than the left one, which implied stricture of right renal artery (Figure 4D). Considering the existence of thrombus, the anticoagulant was changed to rivaroxaban by oral administration for three months. The pathological examination finally confirmed that the tumor was retroperitoneal leiomyosarcoma $(53 \mathrm{~mm} \times 50 \mathrm{~mm}$ 

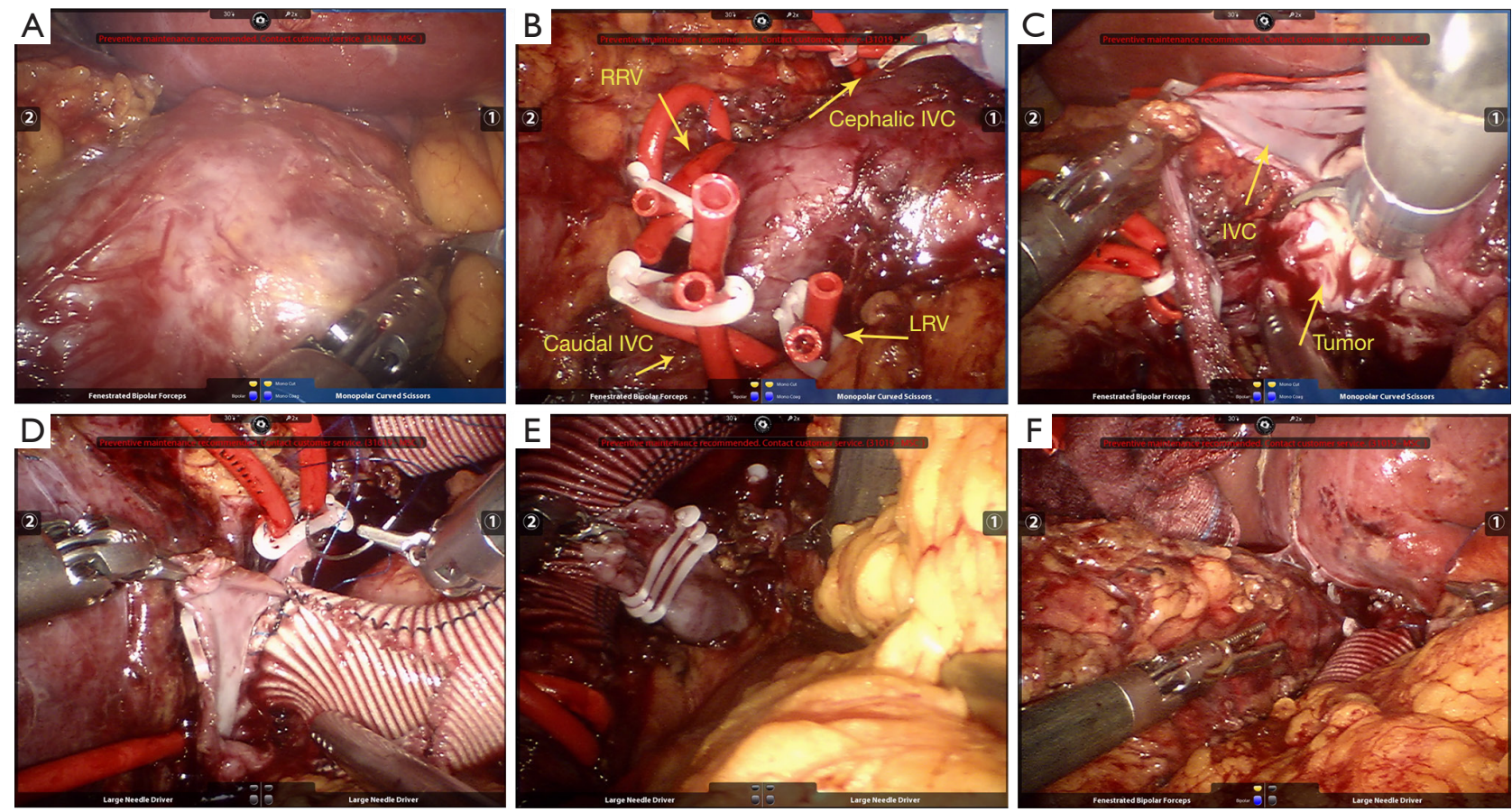

Figure 3 Surgical procedures of the operation. (A) Dissection of adipose tissues and exposure of the mass. (B) Ligation of cephalic IVC, caudal IVC, RRV and LRV. (C) Dissection of tumor from IVC. (D) Jointing of RRV free end to the interposition graft. (E) Isolation of LRV by Hem-o-lok clips. (F) Prevention of the synthetic graft from bowels. IVC, inferior vena cava; RRV, right renal vein; LRV, left renal vein.

$\times 38 \mathrm{~mm}$ size) with grossly negative margins R0 (which means no residual tumor) (Figure $4 E$ ). The patient was discharged 11 days postoperatively and came back in three months for reexamination. Abdominal pain was resolved and vital signs were normal. No lower-extremity edema was found. Ultrasound indicated that blood flow signals were weak in the ePTFE interposition graft. Dynamic renography revealed that GFR of the left kidney was normal with $63.7 \mathrm{~mL} / \mathrm{min}$. However, GFR of the right kidney was significantly decreased to $27.4 \mathrm{~mL} / \mathrm{min}$, which indicated right renal insufficiency. Enhanced MRI was also performed to assess the status of embolus in the graft, which seemed that the filling defection was smaller than before (Figure 4F). Due to the financial stress, the patient returned to the local hospital for further treatments.

\section{Patients and methods}

The patient who underwent the surgery was included from the Wuhan Union Hospital urological center. All procedures performed in studies involving human participants were in accordance with the ethical standards of the institutional and/or national research committee(s) and with the Helsinki Declaration (as revised in 2013). Written informed consent was obtained from the patient.

Hematoxylin and eosin (HE) assay: The tumor sample was fixed with $4 \%$ paraformaldehyde for $12 \mathrm{~h}$. The sections were treatment for deparaffinage in xylene, rehydrated in ethanol, rinsed in distilled water. After fixation with formaldehyde, sections were stained with hematoxylin and eosin followed by dehydration in graded alcohol. The sections were mounted on glass slides previously treated with poly-L-Lysine and examined under a morphometric microscope.

\section{Discussion}

Leiomyosarcomas (LMS) of vascular origins are a rare group of tumors and occur more frequently in women. They are originated from the endothelial smooth muscle and prone to invade adjacent structure by both intra and extraluminal spread (3). Symptoms are determined by the degree of collateral blood flow of the affected segment, the location and the growth rate of tumor (1). Most LMS of vena cava develop on the upper third or the supra-hepatic region and those of mid-portion involves the renal and liver 

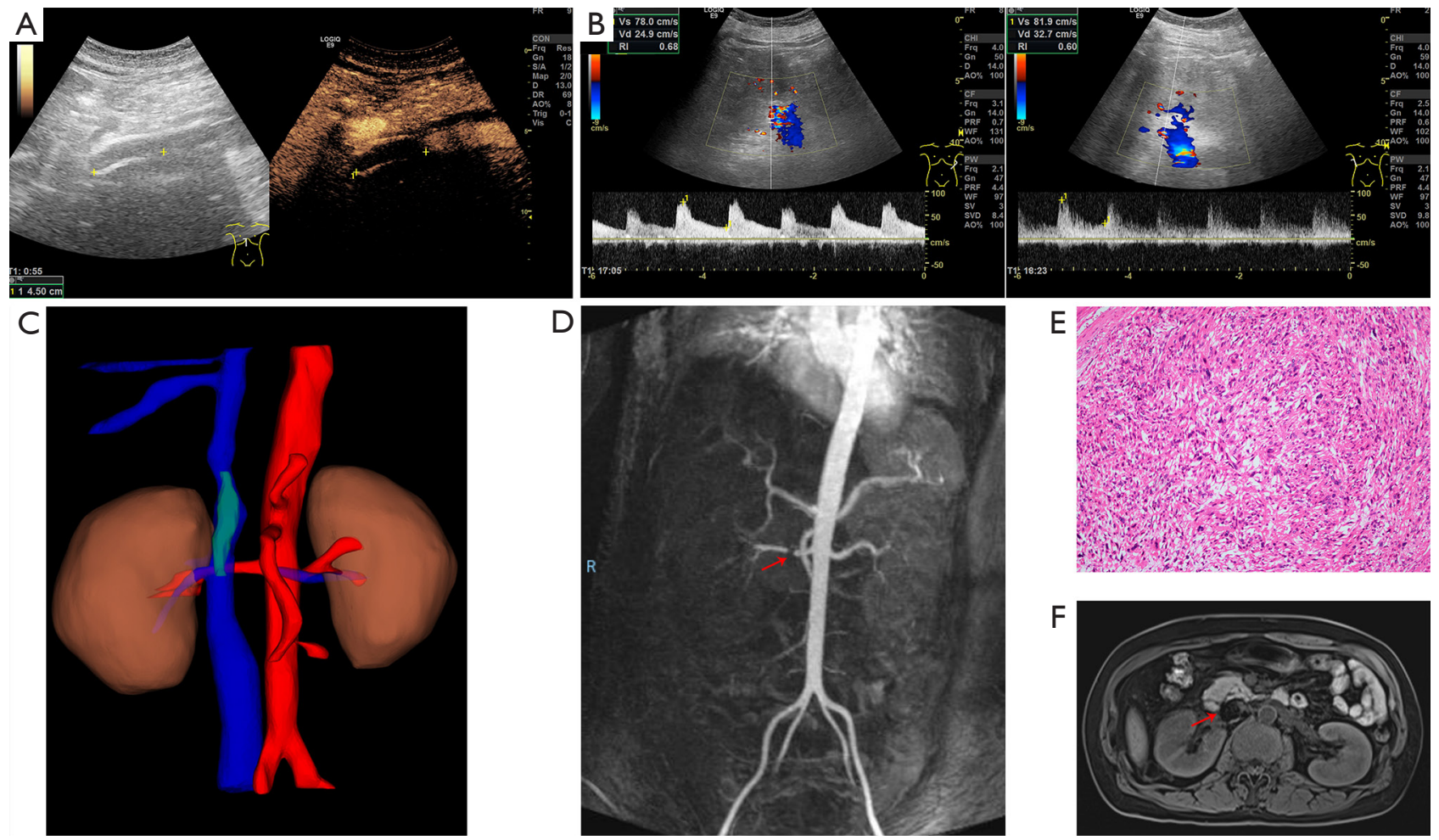

Figure 4 Outcomes of postoperative examination. (A) The ultrasound indicated a $45 \mathrm{~mm}$ filling defection in the upper synthetic graft. (B) The blood flow velocity of renal artery was $78 \mathrm{~cm} / \mathrm{s}$ (left) and $81.9 \mathrm{~cm} / \mathrm{s}$ (right) respectively 6 days postoperatively. (C) Three-dimensional reconstruction of MRI revealed that the graft was partially be occluded by thrombus one week postoperatively. (D) The continuity of right renal artery imaging was interrupted (arrow) one week postoperatively. (E) The pathological examination confirmed that the tumor was retroperitoneal leiomyosarcoma with negative margins R0 (under 100× magnification). (F) Embolus (arrow) in the graft seemed to be smaller 3 months after operation. MRI: magnetic resonance imaging.

veins (10). Local recurrence is reported in $40 \%$ of the cases and metastasis commonly occurs in in liver, kidney, lung and bone (11). Enhanced CT, MRI and cavography are useful to define characteristics of the tumors, invasion status and collateral circulation $(12,13)$.

Adjuvant radiation has been shown to decrease local recurrence and facilitate negative margins, but not to benefit survival for leiomyosarcoma of the IVC (14). Some surgeons prefer preoperative radiation therapy based on the conceptions that IVC is arterialized and handle of this kind of thickened wall is greatly improved (4). Chemotherapy are reserved for patients with postoperative distant metastasis, but the efficacy of chemotherapy, whether preoperative or postoperative, is poorly defined and very limited (15).

Till now, no consensus has been made on the optimal management of the IVC, which include ligation, patch angioplasty, or graft replacement (3). Some authors have advocated for primary or patch repair to minimize lower extremity edema whenever feasible and chosen ligation of the IVC as an alternative choice (16). It has been reported that lower extremity edema occurs in more than $50 \%$ of cases when no IVC reconstruction is undertaken (4). The reason for this is that resection of large retroperitoneal tumors may disrupt venous collaterals, which predisposes patients to developing edema when the IVC is ligated. In our study, the patient, who had transient lower extremity edema symptom and recovered, may benefit from IVC reconstruction. In some cases, patients were undertaken ligation without reconstruction despite complete occlusion of the IVC. This strategy was supported by the facts, which included reduced operative time, prevention of lower extremity deep venous thrombosis, potential lifelong anticoagulation and heart failure from creation of arteriovenous fistulas (4). Although lower extremity edema still existed, but the surgeons believed that this was transitory and well-tolerated phenomenon. Some 
authors favored graft replacement which demonstrated excellent patency rates with minimal complications. One series of patients undergoing ePTFE graft reconstruction demonstrated $75 \%$ late patency rates and no patients were associated with edema (17). Another series of 47 patients with en bloc resection of IVC reported $92 \%$ patency rates with $2 \%$ graft-related complication rate at 5 years (18). In our study, the reason for graft replacement was that the patient may benefit from this due to the young age. Simple ligation without reconstruction will cause venous reflux syndromes in the long term. Even if the thrombosis occurred, it could be rescued by anticoagulation or filter/ stent implantation. Based on our experience, we changed anticoagulants from LMWH to rivaroxaban and asked the patient to follow the prescription for three months. With the improvement of vascular surgery technology, we advocate patch angioplasty or reconstruction whenever possible, as it restores IVC continuity.

As for better exposure of IVC, traditional open approaches prefer a right thoracoabdominal incision, a thoraco-phreno-laparotomy, a combination of median laparotomy and sternotomy and a midline laparotomy incision with a subcostal extension or an extended Kocher maneuver (19). Assistant with auxiliary ports and excellent ergonomics, the robotic laparoscopic system achieves both minimal invasion and better exposure. Till now, one case has been reported about resection of IVC leiomyosarcoma using robot-assisted laparoscopy in a thoracoabdominal approach without vascular management (20). However, in our study, we conducted the operation entirely under robotic surgical systems and performed IVC reconstruction due to the large segment of impairment.

Last but not the least, surgical managements of this study were concluded and may be valuable for surgeons. Before the surgery, the patient was not undertaken biopsy based on the concern that the mass was tightly adhered to the great vessels. Addition to the LMS, the tumor was also suspected to be pheochromocytoma, ganglioneuroma or lymphoma. Though the serum level of epinephrine, norepinephrine, cortisol, adrenocorticotropic hormone and renin angiotensin aldosterone system were all normal, the patient was preventively treated with $\alpha$-blocker and fluid resuscitation one week before surgery. It was revealed from the imaging preoperatively that the porta hepatis was slightly pressed upward with no invasion. Therefore, we chose to retract the liver to expose hepatic pedicle rather than blockade during operation and the liver function exhibited normal levels postoperatively. Owing to the unexposed sight of right renal artery, the accidental injury occurred intraoperatively and thus the patient suffered from right renal insufficiency with lower GFR rate. However, the serum creatinine and total GFR reversed to the normal level just 2 days postoperatively and dynamic renography revealed that GRF of the left kidney was $63.7 \mathrm{~mL} / \mathrm{min}$ three months postoperatively. These results indicated that long time ligation of the left renal vein during surgical procedures was safe and acceptable options for surgeons.

Leiomyosarcomas of IVC present a technical challenge to surgeons. Comprehensive preparation should be made preoperatively to facilitate tumor resection and vascular management. To our knowledge, this is the first case report of a robot-assisted laparoscopic approach for en bloc removal of a large LMS and IVC reconstruction with ePTFE graft. We believe that in specific cases, robotic resection of leiomyosarcoma is acceptable with experienced surgeons and if possible, vascular repairment is favored to restore its continuity.

\section{Acknowledgments}

We appreciated all involved in this study.

Funding: Our study was supported by the Key Research and Development Plan in China (Grant No. 2017YFB1303100), the National Natural Science Foundation of China (Grant No. 82002704, 81927807, 81874090, 81672528, 81773282 \& 81972630), Individual Innovative Research Funding of Union Hospital (Grant No. 02.03.2019156) and the Wuhan Science and Technology Plan Application Foundation Frontier Project (Grant No. 2020020601012247).

\section{Footnote}

Reporting Checklist: The authors have completed the CARE reporting checklist. Available at http://dx.doi.org/10.21037/ tau-20-1523

Conflicts of Interest: All authors have completed the ICMJE uniform disclosure form (available at http://dx.doi. org/10.21037/tau-20-1523). The authors have no conflicts of interest to declare.

Ethical Statement: The authors are accountable for all aspects of the work in ensuring that questions related to the accuracy or integrity of any part of the work are appropriately investigated and resolved. All procedures 
performed in studies involving human participants were in accordance with the ethical standards of the institutional and/or national research committee(s) and with the Helsinki Declaration (as revised in 2013). Written informed consent was obtained from the patient.

Open Access Statement: This is an Open Access article distributed in accordance with the Creative Commons Attribution-NonCommercial-NoDerivs 4.0 International License (CC BY-NC-ND 4.0), which permits the noncommercial replication and distribution of the article with the strict proviso that no changes or edits are made and the original work is properly cited (including links to both the formal publication through the relevant DOI and the license). See: https://creativecommons.org/licenses/by-nc-nd/4.0/.

\section{References}

1. Aguilar IC, Benavente VA, Pow-Sang MR, et al. Leiomyosarcoma of the renal vein: Case report and review of the literature. Urol Oncol 2005;23:22-6.

2. Shmookler BM, Lauer DH. Retroperitoneal leiomyosarcoma. A clinicopathologic analysis of 36 cases. Am J Surg Pathol 1983;7:269-80.

3. Wachtel H, Jackson BM, Bartlett EK, et al. Resection of primary leiomyosarcoma of the inferior vena cava (IVC) with reconstruction: a case series and review of the literature. J Surg Oncol 2015;111:328-33.

4. Daylami R, Amiri A, Goldsmith B, et al. Inferior vena cava leiomyosarcoma: is reconstruction necessary after resection? J Am Coll Surg 2010;210:185-90.

5. Wei TC, Chung HJ, Lin AT, et al. Robot-assisted laparoscopic excision of a retroperitoneal paracaval tumor. J Chin Med Assoc 2013;76:724-6.

6. Berelavichus SV, Zotikov AE, Kriger AG, et al. Twostage combined treatment of leiomyosarcoma of iliac vein using robotic surgery. J Vasc Surg Cases Innov Tech 2019;5:586-8.

7. Vicente E, Quijano Y, Duran H, et al. First case of complete full robotic surgical resection of leiomyosarcoma of the right renal vein. Surg Endosc 2018;32:1072.

8. Trandem K, Aghazadeh MA, Goh AC. Robot-assisted Laparoscopic Resection of Renal Vein Leiomyosarcoma. Urology 2017;103:e1-e2.

9. Saltzman AF, Brown ET, Halat SK, et al. An uncommonly encountered perirenal mass: Robotic resection of renal vein leiomyosarcoma. Can Urol Assoc J 2015;9:E213-6.

10. Brewster DC, Athanasoulis CA, Darling RC.

Leiomyosarcoma of the inferior vena cava. Diagnosis and surgical management. Arch Surg 1976;111:1081-5.

11. Burke AP, Virmani R. Sarcomas of the great vessels. A clinicopathologic study. Cancer 1993;71:1761-73.

12. Dunnick NR. Image interpretation session: 1999. Leiomyosarcoma of the left renal vein. Radiographics 2000;20:269-71.

13. Yuan C, Xiong Z, Shi J, et al. Overexpression of PPT2 Represses the Clear Cell Renal Cell Carcinoma Progression by Reducing Epithelial-to-mesenchymal Transition. J Cancer 2020;11:1151-61.

14. Hines OJ, Nelson S, Quinones-Baldrich WJ, et al. Leiomyosarcoma of the inferior vena cava: prognosis and comparison with leiomyosarcoma of other anatomic sites. Cancer 1999;85:1077-83.

15. Marcheix B, Dambrin C, Muscari F, et al. Leiomyosarcoma of the inferior vena cava. J Chir (Paris) 2003;140:140-8.

16. Hollenbeck ST, Grobmyer SR, Kent KC, et al. Surgical treatment and outcomes of patients with primary inferior vena cava leiomyosarcoma. J Am Coll Surg 2003;197:575-9.

17. Hardwigsen J, Baqué P, Crespy B, et al. Resection of the inferior vena cava for neoplasms with or without prosthetic replacement: a 14-patient series. Ann Surg 2001;233:242-9.

18. Quinones-Baldrich W, Alktaifi A, Eilber F, et al. Inferior vena cava resection and reconstruction for retroperitoneal tumor excision. J Vasc Surg 2012;55:1386-93; discussion 1393.

19. Mastoraki A, Leotsakos G, Mastoraki S, et al. Challenging diagnostic and therapeutic modalities for leiomyosarcoma of inferior vena cava. Int J Surg 2015;13:92-5.

20. Kida K, Terada N, Nakamura E, et al. En bloc removal of a large leiomyosarcoma arising from the inferior vena cava using retroperitoneal laparoscopic dissection of posterior vessels followed by cavectomy in a thoracoabdominal approach. Urol Case Rep 2019;26:100984.

Cite this article as: Cheng G, Ruan H, Yang C, Cao Q, Liang H, Yang X, Jiang G, Zhang X. Robot-assisted laparoscopic retroperitoneal leiomyosarcoma resection with inferior vena cava graft replacement: a case report. Transl Androl Urol 2021;10(5):2133-2139. doi: 10.21037/tau-20-1523 